\title{
Valosin-Containing Protein Gene Mutations: Cellular Phenotypes Relevant to Neurodegeneration
}

\author{
Karen S. Poksay • David T. Madden • Anna K. Peter • \\ Kayvan Niazi • Surita Banwait • Danielle Crippen • \\ Dale E. Bredesen • Rammohan V. Rao
}

Received: 19 November 2010 / Accepted: 22 December 2010/Published online: 20 January 2011

(C) The Author(s) 2011. This article is published with open access at Springerlink.com

\begin{abstract}
Previously, we identified valosin-containing protein (VCP) as a mediator of ER stress-induced cell death. Mutations in the VCP gene including R93, R155, and R191 have been described that manifest clinically as hereditary inclusion body myopathy with Paget's disease of bone and frontotemporal dementia. In addition, other studies have demonstrated that as a consequence of a mutation generated in the second ATP binding domain of VCP (K524A), cells accumulated large cytoplasmic vacuoles and underwent programmed cell death. In order to better understand the biochemical and molecular consequences of the clinically relevant VCP mutations as well as the genetically engineered ATPase-inactive mutant K524A and any relationship these
\end{abstract}

K. S. Poksay · D. T. Madden · A. K. Peter $\cdot$ K. Niazi •

S. Banwait $\cdot$ D. Crippen $\cdot$ D. E. Bredesen $(\bowtie) \cdot R$. V. Rao $(\bowtie)$

The Buck Institute for Research on Aging,

8001 Redwood Blvd,

Novato, CA 94945, USA

e-mail: dbredesen@buckinstitute.org

e-mail: rrao@buckinstitute.org

D. E. Bredesen

University of California, San Francisco,

San Francisco, CA 94143, USA

Present Address:

D. T. Madden

Touro University - California, College of Pharmacy,

Vallejo, CA 94592, USA

Present Address:

A. K. Peter

UC Davis School of Medicine,

Sacramento, CA 95817, USA

Present Address:

K. Niazi

Abraxis Bioscience,

Los Angeles, CA 90025, USA may have to ER stress-induced cell death, we introduced analogous mutations separately and together into the human VCP gene and evaluated their effect on proteasome activity, Huntingtin protein aggregation and ER stress-induced cell death. Our results indicate that the VCP K524A mutant and the triple mutant VCP R93C-R155C-K524A block protein degradation, trigger Huntingtin aggregate formation, and render cells highly susceptible to ER stress-induced cell death as compared to VCPWT or other VCP mutants.

Keywords Valosin-containing protein - Endoplasmic reticulum $\cdot$ ER stress $\cdot$ Programmed cell death

\author{
Abbreviations \\ VCP Valosin-containing protein \\ ER Endoplasmic reticulum \\ FTD Frontotemporal dementia \\ IBMPFD Hereditary inclusion body myopathy with Paget's \\ disease of bone and FTD
}

\section{Introduction}

Accumulation of misfolded proteins induces cellular stress including endoplasmic reticulum stress (ER stress), and prolonged ER stress triggers neuronal apoptotic cell death (Rao and Bredesen 2004; Bredesen et al. 2006; Yang et al. 2010). Studies from multiple laboratories have identified the roles of several ER stress-induced cell death modulators and effectors through the use of biochemical, pharmacological and genetic tools (Kopito 2000; Chen and Gao 2002; Forman et al. 2003; Hashimoto et al. 2003; Katayama et al. 2004; Rao et al. 2004a; Xu et al. 2005; Bredesen et al. 2006; Lindholm et al. 2006; Yoshida 2007). 
In our earlier studies, we used a set of complementary approaches and identified valosin-containing protein (VCP) as one of the mediators of ER stress-induced programmed cell death (Rao et al. 2004a; Bredesen et al. 2006). Valosincontaining protein, also known as p97, is a member of the AAA (ATPases associated with diverse cellular activities) family of ATP binding, homo-oligomeric ATPase proteins and participates in multiple cellular activities, many of which are regulated by the ubiquitin-proteasome (Ub-Pr)mediated degradation pathway (Vale 2000; Wang et al. 2003; Ju et al. 2009; Tresse et al. 2010). VCP consists of an $\mathrm{N}$-terminal domain and two ATPase domains D1 and D2, both of which are required for mediating Ub-Pr degradation (Egerton et al. 1992; Pleasure et al. 1993; Dai et al. 1998; Dai and Li 2001; Hirabayashi et al. 2001; Song et al. 2003; Vandermoere et al. 2006; Zhong and Pittman 2006). VCP also functions as a sensor of abnormally-folded proteins and has been reported to act as a cell death effector in polyglutamine-induced cell death (Hirabayashi et al. 2001; Kobayashi et al. 2002; Wojcik 2002; Doss-Pepe et al. 2003; Zhong and Pittman 2006).

Recently, mutations in the VCP gene including R93, R155, R191, and A232 were described that manifest clinically as hereditary inclusion body myopathy (IBM) with Paget's disease of bone and frontotemporal dementia (FTD; Schroder et al. 2005; Guyant-Marechal et al. 2006; Hubbers et al. 2007; Halawani et al. 2009; Weihl et al. 2009; Custer et al. 2010). This multisystem disorder with autosomal dominant inheritance is associated with disruption of normal VCP function leading to diffuse intracellular accumulation of ubiquitinated proteins that may contribute to the pathogenesis of IBMPFD (Inclusion Body Myopathy associated with $P$ aget's disease of bone and Frontotemporal Dementia; Weihl et al. 2006; Hubbers et al. 2007; Kimonis et al. 2008; Vij 2008; Gitcho et al. 2009; Vesa et al. 2009; Weihl et al. 2009; Custer et al. 2010). Neurons, as well as cells of the muscle, liver, and bone, are affected by these mutations in VCP (Weihl et al. 2006; Gitcho et al. 2009; Vesa et al. 2009; Weihl et al. 2009). In addition, a nonclinical VCP mutant (K524A) located in the 2nd ATP binding domain (D2 domain) induced large cytoplasmic vacuoles and triggered programmed cell death (Hirabayashi et al. 2001; Kobayashi et al. 2002).

In order to elucidate the biochemical and molecular implications of such mutations in the cell we, introduced analogous mutations (separately and together) into the VCP gene to explore their impact on proteasome function, Huntingtin protein aggregation and programmed cell death. Our results demonstrate that, to varying degrees, these VCP mutants: (1) disrupt VCP's normal role in protein homeostasis, (2) trigger the accumulation of ubiquitinated and aggregated proteins that may be deleterious to the cell, and (3) trigger cellular death.

\section{Experimental Procedures}

\section{Cell Culture Conditions and Transfections}

Human embryonic kidney (HEK) 293 T cells were cultured in DMEM containing 10\% FBS and 1\% penicillin/ streptomycin. Cells were maintained at $37^{\circ} \mathrm{C}$ and $5 \%$ $\mathrm{CO}_{2}$. Transient transfection was performed as previously described (Rao et al. 2001). In brief, $2 \times 10^{6}$ cells were seeded into $10-\mathrm{cm}$ dishes and transfected 1 day later with $6 \mu \mathrm{g}$ of the specified construct using a ratio of $1 \mu \mathrm{g}$ DNA:5 $\mu$ l of Lipofectamine 2000 transfection reagent (Invitrogen, Carlsbad, CA). The transfection efficiency using these conditions was typically about $65-75 \%$ as determined by GFP transfection efficiency. Alternatively, transfections for flow cytometry or immunocytochemistry were carried out in multi-well dishes as specified in the figures.

Generation of Constructs Encoding VCP Mutants Unless otherwise stated, all molecular biology enzymes were purchased from New England Biolabs (Ipswich, MA). Expression plasmids encoding VCPWT and VCP K524A in the pCMX backbone were kindly provided by Dr. A. Kakizuka (Hirabayashi et al. 2001; Rao et al. 2004b). The cDNAs encoding wild-type (WT) and K524A VCP were transferred from the parent vectors by KpnI digestion and ligation into the KpnI site of pcDNA3.1/Myc-His-A (Invitrogen, Carlsbad, CA) and resultant ligation mixtures transformed into DH5 $\alpha$ competent cells (Invitrogen). Clones possessing the VCP cDNAs in the correct orientation with regards to the cytomegalovirus promoter were identified by PCR using the Failsafe DNA polymerase (Epicentre Biotechnologies, Madison, WI). The resulting pcDNA3.1/Myc-His-A/VCP construct was used as a template for the addition of a BsmBI site containing a partially destroyed HindIII site, a novel SacII site, and an N-terminal FLAG tag sequence using PCR and the oligonucleotides 5' VCP FLAG and VCP HindIII Rev (Table 1). The amplified product was digested with BsmBI and HindIII, ligated into HindIII-digested pcDNA3.1/ Myc-His-A/VCP and pcDNA3.1/Myc-His-A/VCP K524A, and transformed colonies were interrogated by PCR to determine insert orientation. Resulting constructs named pcDNA3.1/Myc-His-A/VCPWT-FLAG or pcDNA3.1/ Myc-His-A/VCPK524A-FLAG were evaluated by restriction analysis to confirm that the $5^{\prime} \mathrm{KpnI}$ site borrowed from the 
Table 1 Primer design for the various VCP mutant constructs

\begin{tabular}{ll}
\hline R93C & REV: TCCTAGGCGAACGCAGAGGTTATTCCG \\
& FOR: CGGAATAACCTCTGCGTTCGCCTAGGA \\
VCP-HindIII & REV: GGATGGGCCGATAAGCTTCCAGGAAGTACG \\
& FOR: CGTACTTCCTGGAAGCTTATCGGCCCATCC \\
R155C & REV: ACGCATCCCACCGCAGACAAGAAAAAT \\
& FOR: QTTTTTCTTGTCTGCGGTGGGATGCGT \\
VCP-NheI & REV: TCCTTTATCTGAGCTAGCTGCTTCCTGCAA \\
& FOR: TTGCAGGAAGCAGCTAGCTCAGATAAAGGA
\end{tabular}

5'VCP-FLAG*- GAATTCCGTCT CA AGCT $C C G C G G$ ATGGATTA CAAGGATGACGACGATAAG ATG GCCTCTGGAGCCGATTCAA

*From left: BsmBI (italicized), HindIII complementary overhang (bold), SacII site (italicized \& underlined), start methionine and FLAG-tag corresponding codons (bold \& underlined)

original pCMX backbone had been successfully removed, and further confirmed by DNA sequencing.

As a result of this cloning strategy, it became possible to separate the three desired mutations into individual modular domains through the use of unique restriction sites. For instance, the $5^{\prime} \sim 450 \mathrm{bp}$ of VCP containing amino acid residue 93 were restricted by SacII and HindIII, the amino acid R155 was flanked by the sites HindIII on the $5^{\prime}$ and NheI on the $3^{\prime}$ sides, and the amino acid K524 was flanked by the sites NheI on the $5^{\prime}$ and the remaining KpnI site from the original pCMX construct on the $3^{\prime}$ side. The R93C mutant was created using pcDNA3.1/Myc-His-A/VCPWTFLAG as a template for PCR amplification to generate the $5^{\prime} \mathrm{R} 93 \mathrm{C}$ reaction product using the oligonucleotides $5^{\prime} \mathrm{VCP}$ FLAG and R93C REV and the 3' R93C reaction product using oligonucleotides R93C FOR and VCP HindIII REV (Table 1). Both overlapping products were gel-purified, combined, and amplified using the external $5^{\prime}$ VCP FLAG and 3' VCP HindIII REV oligonucleotides and the resulting product was digested by SacII and HindIII and ligated into the corresponding SacII and HindIII sites of pcDNA3.1/ Myc-His-A/VCPWT-FLAG to create the construct pcDNA3.1/Myc-His-A/VCPR93C-FLAG. Similarly, the R155C mutant was created using pcDNA3.1/Myc-His-A/ VCPWT-FLAG as a template for PCR amplification to generate the $5^{\prime} \mathrm{R} 155 \mathrm{C}$ reaction product using the oligonucleotides VCP HindIII FOR and R155C REV and the 3' $\mathrm{R} 155 \mathrm{C}$ reaction product using oligonucleotides $\mathrm{R} 155 \mathrm{C}$ FOR and VCP NheI REV (Table 1). Both overlapping products were gel purified, combined, and amplified using the external VCP HindIII FOR and VCP NheI REV oligonucleotides and the resulting product was digested by HindIII and NheI and ligated into the corresponding HindIII and NheI sites of pcDNA3.1/Myc-His-A/ VCPWT-FLAG to create the construct pcDNA3.1/
Myc-His-A/VCPR155C-FLAG. VCPDM (double mutant R93C, R155C) and VCPTM (triple mutant R93C, R155C, K524A) were created by subcloning the desired fragments into the appropriate locations using the matching unique restriction sites described above in a modular fashion. All constructs were verified by DNA sequencing and Western blot analyses were performed to verify protein expression.

Other than some changes in the total number of amino acids and the N-terminal tag, the Huntingtin protein $(\mathrm{Htt})$ expression constructs (gift from Dr L.M. Ellerby) used in these studies included the GFP-tagged normal N-terminal Htt fragment Htt23Q-(1-552) and the expanded polyQ-Htt144Q$(1-552)$ that have been previously described (Igarashi et al. 2003; Schilling et al. 2006). The sequences of all constructs were confirmed by DNA sequencing, and Western blot analyses were performed to verify protein expression.

\section{Cell Extracts, Cell Fractionation and Western Blotting} Whole cell extracts were prepared as described earlier (Rao et al. 2001). Briefly, cells were collected and resuspended in cell lysis buffer (500 mM HEPES, pH 7.6, $250 \mathrm{mM}$ $\mathrm{NaCl}, 0.1 \%$ Nonidet P-40, $5 \mathrm{mM}$ EDTA) containing complete-mini protease inhibitors (Roche). Resuspended cells were sonicated three times for $30 \mathrm{~s}$ each, and unbroken cells and membrane debris were removed by centrifugation at $4^{\circ}$ for $15 \mathrm{~min}$ at $20,000 \times \mathrm{g}$. Cell-free extracts and microsomes were prepared as previously described (Rao et al. 2001, 2002, 2004b, 2006). Briefly, the 20,000 $\times g$ supernatant that lacks whole cells, nuclei and mitochondria was recentrifuged at $400,000 \times g$ for $1 \mathrm{~h}$ at $4{ }^{\circ} \mathrm{C}$ for the preparation of microsomes. The resulting supernatant (S) contains the soluble cytosolic fraction and the pellet $(\mathrm{P})$ represents microsomes (ER membranes and lumen proteins) as well as Golgi membranes. Typically, $100 \mu \mathrm{g}$ of cell extract was loaded for SDS-PAGE and Western blot analysis as previously described (Rao et al. 2001, 2002, 2004b, 2006). PVDF membranes were probed with a 1:1,000 dilution of mouse anti-VCP monoclonal antibody (BD-Transduction Laboratories) and a 1:500 dilution of rabbit anti-GRP78 polyclonal antibody (Stressgen-Assay Designs Life Sciences) as a standard marker protein for ER.

Cell Death Analysis by Flow Cytometry Flow cytometry analysis was performed as previously described (Egger et al. 2007). Briefly, attached and floating cells were collected by low-speed centrifugation after gentle trypsinization and stained with $3 \mu \mathrm{g} / \mathrm{ml}$ GFP-Annexin- $\mathrm{V}$ and $2.5 \mu \mathrm{g} / \mathrm{ml}$ propidium iodide in Annexin- $\mathrm{V}$ binding buffer (10 mM Hepes/NaOH, pH 7.4, $140 \mathrm{mM} \mathrm{NaCl}, 2.5 \mathrm{mM}$ $\mathrm{CaCl}_{2}$ ) and incubated at room temperature for $15 \mathrm{~min}$. 
Cells were analyzed on a BD LSR flow cytometer (BD Biosciences, San Jose, CA) where annexin-V (dying) and propidium iodide (dead) positive cells were quantified. Data were processed using CellQuest Pro (BD Biosciences).

Analysis of Ubiquitin-Proteasome Function by Transfection with GFPu To investigate the specific role of VCP and its mutants in protein aggregation and ubiquitin-proteasome system function, we made use of a GFPu reporter construct kindly provided to us by Dr. Ron Kopito (Stanford University). The reporter consists of a short degron CL1 sequence fused to the $\mathrm{COOH}$ terminus of green fluorescent protein; this fusion protein is referred to as GFPu. By appending this short sequence to the C-terminus of GFP, the protein becomes unstable and is constitutively degraded by the proteasome. In the present study, 293T cells were transfected with GFPu cDNA together with VCPWT or its various mutants at a ratio of $1: 3$, respectively. After $24 \mathrm{~h}$, $\mathrm{GFPu}$ immunofluorescence accumulation was quantified by flow cytometry (Bence et al. 2001; Bence et al. 2005; Egger et al. 2007).

Immunocytochemistry HEK 293T cells were plated at 60$75 \%$ confluency on glass coverslips. Twenty-four hours after plating the cells, transfections were carried out as mentioned above and following the manufacturer's protocol (Invitrogen). Twenty-four hours after transfection, samples were treated with or without $1 \mu \mathrm{M}$ thapsigargin for 24 hours. Cells were fixed in buffered 4\% paraformaldehyde and permeabilized in $0.2 \%$ Triton in PBS. For the detection of VCP, cells were stained with mouse monoclonal antiVCP (BD-Transduction Laboratories, 1:200) and Alexa Fluor 488 donkey anti-mouse IgG $(4 \mu \mathrm{g} / \mathrm{ml}$, Invitrogen, Carlsbad, CA) as the secondary antibody. For the detection of GRP78, cells were stained with rabbit polyclonal anti-GRP78 (Santa Cruz Biotechnology, 1:100) and Alexa Fluor 555 donkey anti-rabbit IgG $(2 \mu \mathrm{g} / \mathrm{ml}$, Invitrogen, Carlsbad, CA). Nuclei were counterstained using VectaShield Mounting Medium with DAPI (Vector Laboratories, Burlingame, CA).

For confocal microscopy studies involving VCP and $\mathrm{Htt}$ constructs, a similar staining procedure was followed. Following transfection of $293 \mathrm{~T}$ cells for $48 \mathrm{~h}$ with VCPWT or mutants and Htt-23Q or Htt-144Q cDNA, cells were fixed in $4 \%$ paraformadehyde in PBS for $20 \mathrm{~min}$. After three PBS washes, cells were permeabilized with $0.2 \%$ Triton in PBS for $15 \mathrm{~min}$ and serially stained first for Huntingtin and then for VCP in the following manner. Cells were blocked for $30 \mathrm{~min}$ in $5 \%$ normal donkey serum (NDS) in PBS. Mouse IgG anti-Huntingtin protein
(MAB2166, Chemicon) was diluted 1:50 in 2.5\% NDS in PBS and incubated overnight at $4^{\circ} \mathrm{C}$. Cells were washed three times in PBS and incubated in secondary antibody (Alexa Fluor 555 donkey anti-mouse IgG, $2 \mu \mathrm{g} / \mathrm{ml}$, Invitrogen) diluted in $2.5 \%$ NDS for $1 \mathrm{~h}$ at room temperature (RT). Cells were then washed three times in PBS and re-blocked in 5\% NDS with $0.3 \%$ Triton in PBS for $30 \mathrm{~min}$. Rabbit $\mathrm{IgG}$ anti-VCP (BD-Transduction Laboratories) was diluted $1: 50$ in $0.3 \%$ Triton in PBS and incubated overnight at $4{ }^{\circ} \mathrm{C}$. Cells were then washed three times in PBS and incubated in secondary antibody (Alexa Fluor 488 donkey anti-rabbit $\mathrm{IgG}, 4 \mu \mathrm{g} / \mathrm{ml}$, Invitrogen) diluted in $0.3 \%$ triton in PBS for $1 \mathrm{~h}$ RT. Cells were again washed three times with PBS, air dried and mounted with Prolong Gold with DAPI (Invitrogen P36931). Confocal images were acquired using a Zeiss LSM510 NLO with Argon $488 \mathrm{~nm}, 543 \mathrm{~nm} \mathrm{HeNe}$ and Coherent Chameleon XR 2p laser with appropriate excitation and emission filters. For counting Htt aggregates, three random fields in each sample were selected and the cells containing aggregates were counted with Bitplane Imaris 6.4 using the Spots module. From the GFP-positive cells in a given field, total number of aggregates were counted and averaged among the three replicate fields containing at least 300 cells/sample. Units are expressed as the average number of aggregates/cell and are determined by quantifying the average number of GFP aggregates per GFP-positive cells.

Statistical Analysis All experiments were performed at least three times unless otherwise indicated. Data were statistically analyzed using one-way ANOVA followed by between group comparisons using the Newman-Keuls Multiple Comparison Test (GraphPad Prism, San Diego, $\mathrm{CA}$ ). Results are expressed as mean $\pm \mathrm{SEM}$, and a value of $p<0.05$ was considered statistically significant.

\section{Results}

Expression of VCP Protein in ER Stress-Induced Cells VCP functions as a sensor of abnormally folded proteins and has been reported to act as a cell death effector (Wojcik 2002; Rao et al. 2004b; Ju et al. 2008; Gitcho et al. 2009). The naturally occurring VCP mutants $\mathrm{R} 93 \mathrm{C}$ and $\mathrm{R} 155 \mathrm{C}$ are associated with frontotemporal dementia and inclusion body myopathy (Kimonis et al. 2008; Gitcho et al. 2009). To understand how these mutations might affect VCP function, we designed analogous mutations separately (single mutants) and together (double or triple mutants) 
into the human VCP gene to explore their impact on cellular homeostasis. As shown in Fig. 1a, the expression levels of VCPWT and the various mutant forms were very similar in HEK 293T cells. To examine the subcellular localization of VCPWT and its mutant forms before and/or after ER stress induction, HEK 293T cells were transfected with the various VCP constructs and either left untreated or treated with $1.0 \mu \mathrm{M}$ thapsigargin. Microsomes $(400,000 \times g$ pellet $(\mathrm{P}))$ and the $400,000 \times g$ supernatant $(\mathrm{S})$ isolated from cell-free cytosolic extracts that lack whole cells, nuclei, and mitochondria were subjected to SDS-PAGE and Western blot analysis. As shown in Fig. 1b, both VCPWT and its mutant forms again displayed a similar level of expression. Whereas VCP and its mutant forms were predominantly present in the cytosolic fraction (S) before ER stress, there was increased presence in the microsomal fraction $(\mathrm{P})$ with thapsigargin treatment. A similar pattern of expression was also seen in cellular extracts isolated from cells treated with $2.5 \mu \mathrm{M}$ brefeldin or $0.1 \mathrm{mg} / \mathrm{ml}$ tunicamycin (data not shown).

It is not clear from Fig. 1 if cellular stress is triggering induction of VCP protein expression and/or causing cellular redistribution of VCP. Earlier studies have shown that cellular stress triggers both transcriptional induction and/or cellular relocalization of several ER-associated proteins responsible for the ER-associated degradation (ERAD) pathway including GRP78, VCP and HSP90 that direct the ubiquitin-mediated degradation of a variety of ERassociated misfolded and normal proteins (Wojcik 2002; Mimnaugh et al. 2006; Ju et al. 2009).

The localization of VCP was also investigated using immunocytochemistry on untreated and thapsigargintreated cells using a VCP-specific antibody. As shown in Fig. 1c, the antibody to VCP stained within the cytosolic compartment with a somewhat diffuse granular pattern in the control cells (green). GRP78, a marker protein for ER, demonstrated punctate staining predominantly in the ER (red). Virtually no co-localization of GRP78 and VCP occurred under control conditions (merged). After treatment with $1.0 \mu \mathrm{M}$ thapsigargin for $24 \mathrm{~h}$, VCP staining was more intense, and this increased staining was associated with the ER. GRP78 staining also became more intense, appeared granular predominantly in the ER, and also displayed some diffuse distribution in the cytoplasmic region. Following thapsigargin treatment, approximately half the green fluorescent volume (VCP) in the treated sample was found to co-localize with GRP78. A similar pattern of cellular localization before and after ER stress was seen in cells transfected with the VCP mutants (data not shown). These data suggest that ER stress not only increases the expression of VCP but also triggers a subpopulation of
VCP to associate with the microsomal compartment as described earlier (Mimnaugh et al. 2006).

Expression of VCP Mutants Triggers Cell Death Earlier reports indicated that VCP is a major mediator of ER stress-induced apoptosis and the inhibition of VCP expression resulted in a reduction of ER stress-induced cell death (Rao et al. 2004a). The naturally-occurring and clinically-described VCP mutants R93C, R155C, and R191A demonstrate a cellular phenotype in which their mediation of cell death is increased (Kimonis et al. 2008; Gitcho et al. 2009). To better understand this phenomenon, we transfected HEK 293 T cells with single, double, or triple mutants of VCP and treated the cells with $2.5 \mu \mathrm{M}$ thapsigargin for $48 \mathrm{~h}$. As shown in Fig. 2, flow cytometry analysis revealed that treatment with thapsigargin led to approximately $30 \%$ cell death in cells transfected with VCPWT, R93C, R155C or DM (double mutant R93C, R155C). However, cells transfected with VCP K524A or TM (triple mutant R93C, R155C, K524A) showed an increased susceptibility to cell death triggered by thapsigargin; over $40 \%$ of the cells transfected with VCP K524A or TM were GFPAnnexin-Vpositive with thapsigargin treatment and had significantly more cell death than did VCPWT.

The VCP K524A mutant has previously been shown to induce large cytoplasmic vacuoles and eventually trigger cell death (Hirabayashi et al. 2001; Kobayashi et al. 2002; Manno et al. 2010). While both the VCP K524A single mutant and the VCP TM (triple mutant that includes the K524A mutation) produced a clear effect on cell death, the clinically relevant FTD-associated mutants alone did not. Earlier reports on other chaperone proteins possessing an intrinsic ATPase activity demonstrate that abolishing the ATPase activity either through base mutations or specific inhibitors causes ER disruption, prevents substrate binding, inhibits chaperone function and triggers cytostasis or cell death (Zhang and Kaufman 2006; Egger et al. 2007; Mishra et al. 2007; Seki et al. 2007; Gitcho et al. 2009; Ju et al. 2009). Thus, it appears that inactivation of the ATPase activity of VCP (K524A and VCP TM) may disrupt a broad spectrum of cellular functions through its binding to specific substrates.

VCP Regulates Ubiquitin-Proteasome Function p97/VCP is believed to be a cellular sensor protein that detects abnormal protein accumulation in the cell and promotes their degradation through the ubiquitin-proteasome system (UPS) in a phenomenon called ERAD (Weihl et al. 2006; Zhong and Pittman 2006; Vij 2008; Gitcho et al. 2009; Yang et al. 2010). To better understand how FTD-related 
A

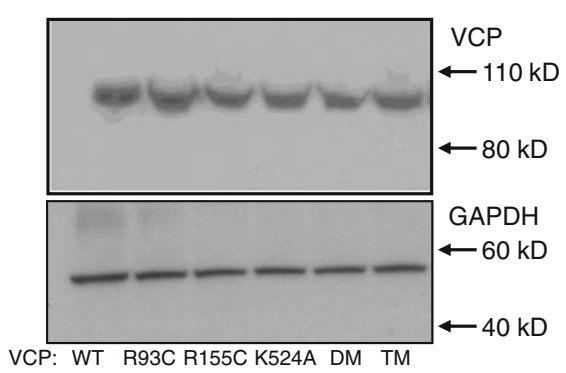

B

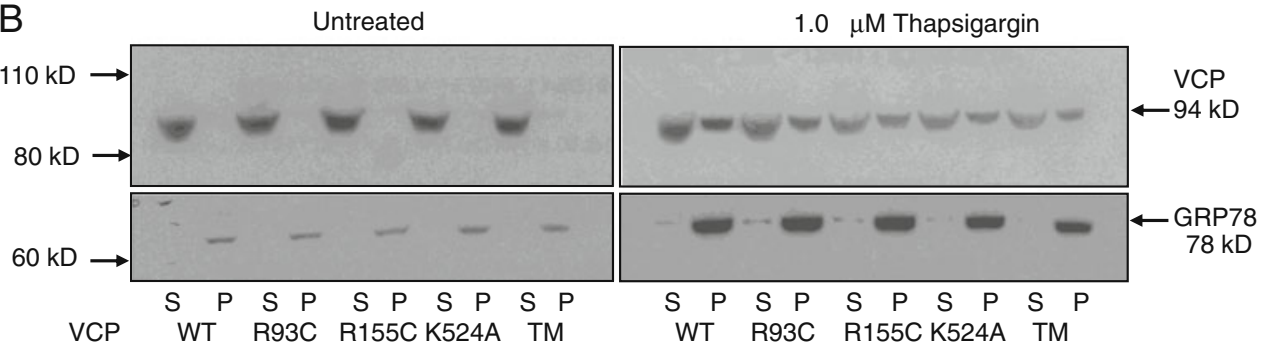

C

Untreated

Thaps

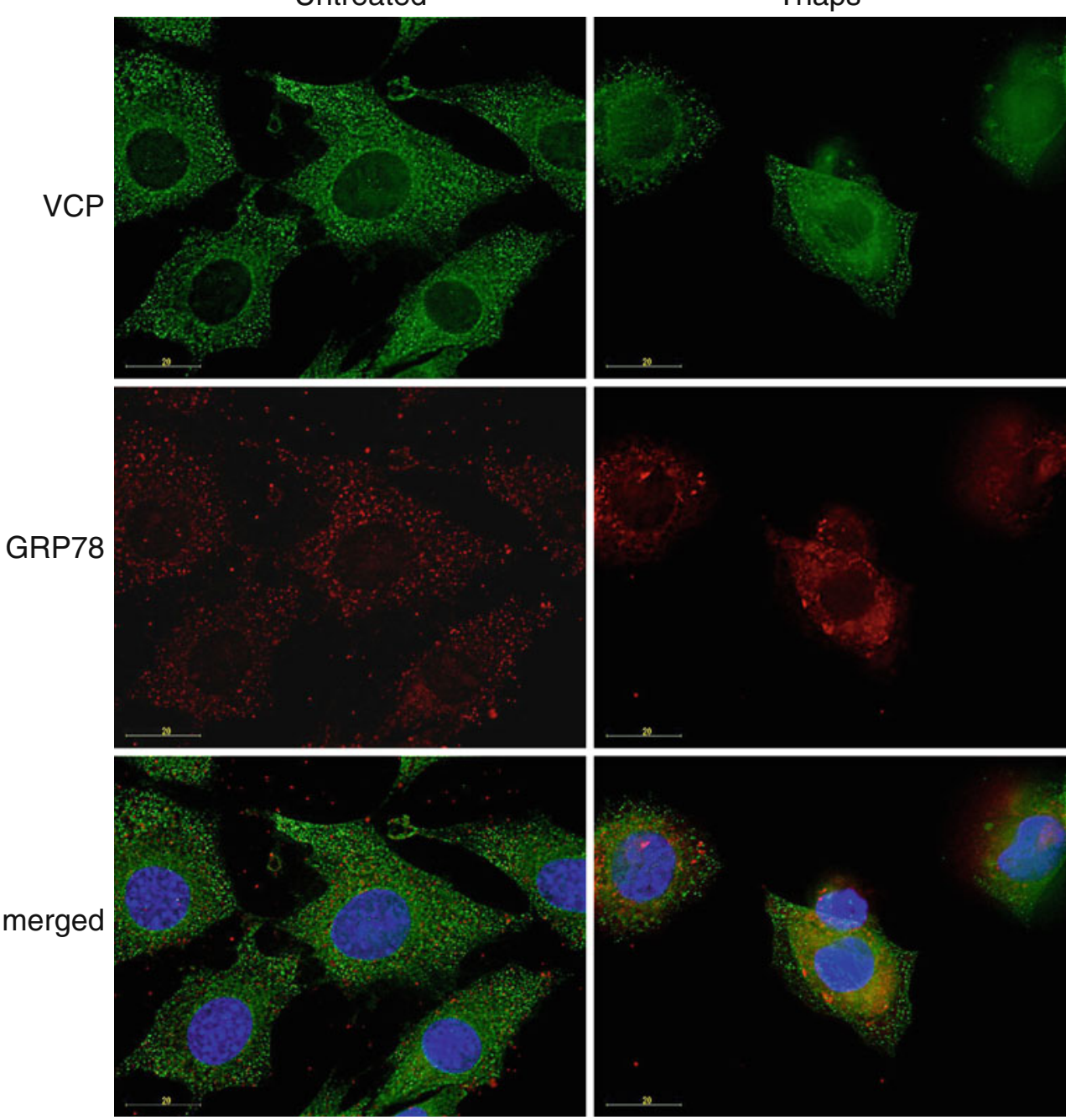


Fig. 1 Expression and subcellular localization of VCP before and after ER stress. a Western blot of whole cell extracts prepared from HEK293T cells transfected with VCPWT, R93C, R155C, K524A, R93C/R155C (double mutant; DM) or R93C/R155C/K524A (triple mutant; TM) cDNA construct as described in "Experimental Procedures". Samples $(100 \mu \mathrm{g}$ protein) were analyzed by SDS-PAGE followed by Western blotting with an anti-VCP antibody. b $293 \mathrm{~T}$ cells were transfected with VCPWT, R93C, R155C, K524A, or the TM cDNA construct. After $24 \mathrm{~h}$, cells were either left untreated or treated with $1.0 \mu \mathrm{M}$ thapsigargin (Thaps) for $24 \mathrm{~h}$. Microsomes were isolated from cell extracts as described in "Experimental Procedures" and $100 \mu \mathrm{g}$ protein from the $400,000 \times g$ supernatant $(S)$ or microsomal $(P)$ fractions was subjected to SDS-PAGE and Western blotting. Membranes were probed with an anti-VCP antibody or anti-GRP78 antibody that served as a standard marker protein for ER. c Immunofluorescent staining for VCP and GRP78 in untreated or $1.0 \mu \mathrm{M}$ thapsigargin-treated HEK293T cells. $24 \mathrm{~h}$ after treatment, cells were fixed in buffered $4 \%$ paraformaldehyde. For the detection of VCP, cells were stained with a mouse monoclonal anti-VCP antibody as primary antibody and 488-conjugated donkey antimouse IgG as the secondary antibody (green). For the detection of GRP78, cells were stained with rabbit polyclonal anti-GRP78 antibody as primary antibody and 555-conjugated donkey antirabbit IgG as the secondary antibody $(r e d)$. Nuclei are counterstained using VectaShield Mounting Medium with DAPI

VCP mutations might dysregulate the UPS, we made use of a fluorescent GFPu protein construct. By appending a short degron sequence CL1 to the C terminus of GFP, the GFPu protein is constitutively degraded by the proteasome (Bence et al. 2005). Thus, GFPu is unstable as compared to normal GFP, but in the presence of the selective proteasome inhibitors lactacystin or ALLN (Nacteyl-leucine-leucinenorleucinal), the UPS-mediated deg-

radation of GFPu is blocked, leading to increased steadystate GFPu levels (Bence et al. 2005; Egger et al. 2007).

To assess the effect of the VCP mutants on GFPu stability/accumulation, HEK 293T cells were co-transfected with GFPu and VCPWT or its various mutants and analyzed for GFPu fluorescence by flow cytometry. As shown in Fig. 3, while cells transfected with VCPWT, R93C, R155C or DM had relatively low levels of GFPu fluorescence, cells transfected with VCP K524A or the TM resulted in an accumulation of GFPu and substantially increased $\mathrm{GFPu}$ fluorescence. GFPu fluorescence was approximately 1.5 -fold higher in cells transfected with VCP K524A or the TM than that of VCPWT, suggesting that the presence of VCP K524A led to a partial inhibition of the UPS. The results also suggest that GFPu degradation is VCP dependent and requires an intact D2-ATPase domain (Kimonis et al. 2008; Gitcho et al. 2009; Halawani et al. 2009).

Effect of VCP Mutants on Poly (Q) Huntingtin Protein Degradation Since expression of VCP K524A and the K524A-containing triple mutant led to inhibition of the UPS, we looked to see whether VCP and its mutants alter poly(Q) huntingtin protein degradation and turnover, with the rationale for these studies being that VCP has been shown to interact with huntingtin as well as other polyglutamine proteins (Kobayashi et al. 2002; Ju et al. 2008; Yang et al. 2010). We co-expressed VCP and its mutants in HEK 293T cells together with GFP-tagged Htt-23Q or expanded Htt-144Q and also quantified the number of Htt-poly

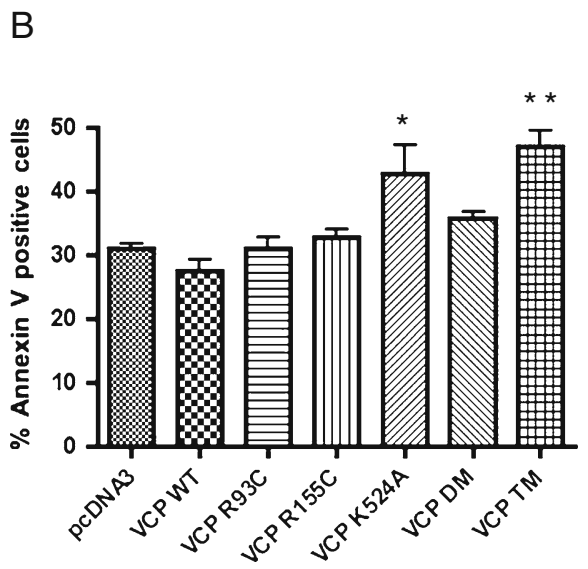

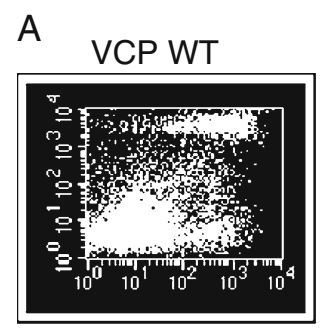

VCP DM
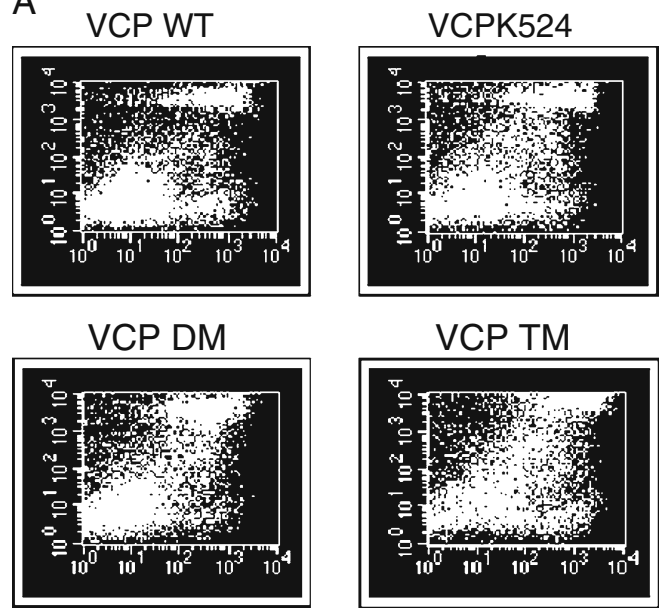

Fig. 2 Expression of VCP mutants triggers cell death. HEK293T cells were transfected with either VCPWT, R93C, R155C, K524A, DM (R93C, R155C) or TM (R93C, R155C, K524A) cDNA construct and treated for $48 \mathrm{~h}$ with $2.5 \mu \mathrm{M}$ thapsigargin. Cells were gently lifted, stained with GFP-annexin-V and propidium iodide and assessed for cell death by flow cytometry as described in "Experimental Procedures". a Representative dot plots from fluorescently-stained cells are shown with annexin- $\mathrm{V}$ positive dying and dead cells occupying the area on the right side of the plot. b Bar graph represents the percent of annexin-V positive cells. Data (mean \pm SEM) are from three independent experiments analyzed by one-way ANOVA followed by NewmanKeuls Multiple Comparison Test $\left({ }^{*} p<0.01,{ }^{* *} p<0.001\right.$ as compared to VCP WT) 


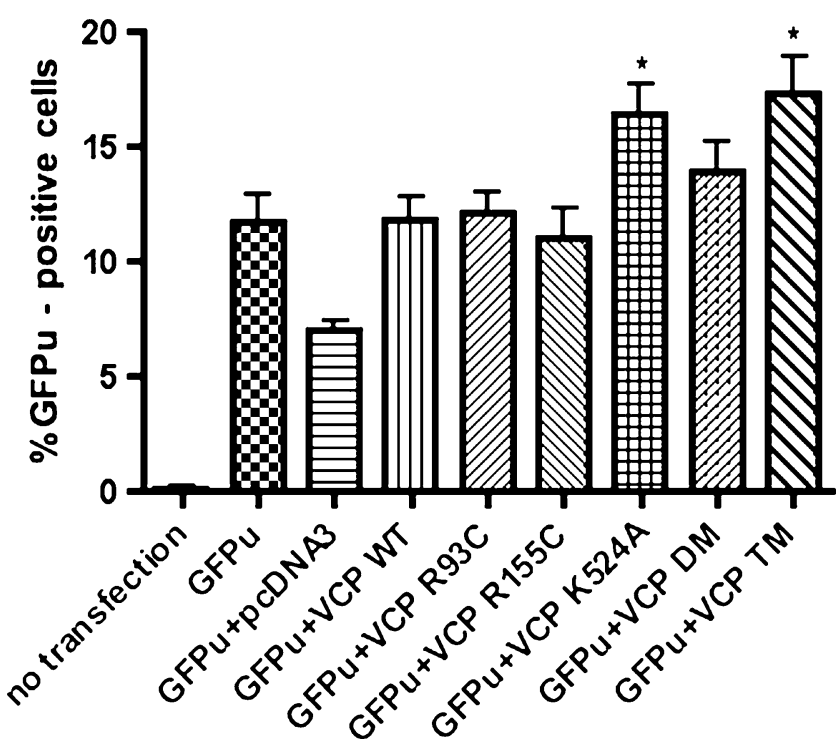

Fig. 3 Analysis of proteasome activity. Proteasome activity was determined by measuring the accumulation of green fluorescence in cells transiently transfected with GFPu together with the VCP mutants. HEK 293T cells were transfected with VCPWT, R93C, R155C, K524A, DM (R93C, R155C) or TM (R93C, R155C, K524A) cDNA construct together with GFPu. Twenty-four hours after transfection, accumulation of GFPu fluorescence was measured by flow cytometry. The bar graph represents the percent of cells positive for $\mathrm{GFPu}$ fluorescence. Data (mean $\pm \mathrm{SEM}$ ) are from two independent experiments analyzed by one-way ANOVA followed by NewmanKeuls Multiple Comparison Test $(* p<0.05, * * p<0.01$ as compared to VCPWT)

(Q)-associated aggregates. As shown in Fig. 4a, cells cotransfected with normal length Htt-23Q exhibited far fewer aggregates than the expanded Htt-144Q in the presence of either VCPWT or the mutant forms. However, in the presence of VCP K524A, DM or TM, even normal length Htt-23Q had a high tendency to aggregate into cytoplasmic and nuclear inclusions while cells expressing the expanded Htt-144Q displayed aggregates that were nearly two-fold higher (Fig. 4b), further suggesting a role for VCP in UPSmediated protein degradation (Bence et al. 2001; Kobayashi et al. 2002). Earlier reports have shown that Poly(Q)containing proteins such as huntingtin and ataxin bind VCP and decrease retrotranslocation of substrates from the ER resulting in decreased degradation of ERAD substrates (Weihl et al. 2006; Zhong and Pittman 2006; Vij 2008; Gitcho et al. 2009; Yang et al. 2010). This raises the possibility that excessive binding between VCP and poly (Q) proteins over an extended period of time or during times of increased cellular stress shifts a physiological regulation to a pathophysiological uncoupling of ERAD which may then contribute to disease pathogenesis (Hirabayashi et al. 2001; Higashiyama et al. 2002; DossPepe et al. 2003; Boeddrich et al. 2006; Zhong and Pittman 2006).

\section{Discussion}

VCP is involved in three distinct processes all related to cell death and neurodegeneration: (1) VCP is required for ER stress-induced programmed cell death where it functions as a sensor of abnormally folded proteins (Rao et al. 2004b); (2) naturally occurring VCP mutations are associated with the neurodegenerative disease IBMFTD when they are part of a syndrome that includes FTD, Paget's disease of bone, and inclusion body myopathy (Schroder et al. 2005; Forman et al. 2006; Guyant-Marechal et al. 2006; Hubbers et al. 2007; Halawani et al. 2009; Weihl et al. 2009; Custer et al. 2010); and (3) our present results also indicate that VCP mutations, and in particular the K524A ATPase mutant, perturb normal VCP functions, including altering the ubiquitin-proteasome system, triggering abnormal protein aggregation and accumulation leading to increased cell death.

VCP has two ATPase domains (D1 and D2) and belongs to the AAA class of proteins that also includes NSF ( $N$-ethylmaleimide-sensitive fusion protein), Hsp104p (heat shock protein 104) and p97/Cdc48p (ortholog of VCP in yeast) (Vale 2000; Song et al. 2003; Halawani et al. 2009). Intact ATPase activity is required for various biological functions of VCP including an intrinsic chaperone activity and binding to ubiquitinated proteins and presenting them to the UPS for degradation (Song et al. 2003; Vij 2008; Gitcho et al. 2009; Manno et al. 2010). While the D1-ATPase domain forms a relatively stable ring and holds the VCP hexamer together throughout the ATPase cycles, the conformational changes induced by the nucleotide binding is mainly attributed to the D2-ATPase domain (Song et al. 2003).

All the clinical mutations of VCP associated with the neurodegenerative disease IBMFTD described thus far reside within the $\mathrm{N}$-terminus and D1-ATPase domain, regions that are proposed to be involved in substrate binding and cofactor association (Watts et al. 2004; Ju et al. 2009). Biochemical characterization of these VCP mutations indicates that not only do they form stable hexamers but they also have increased ATPase activity and efficiently bind to co-chaperone proteins that are essential for UPS function (Hubbers et al. 2007; Halawani et al. 2009; Ju et al. 2009). The increased ATPase activity may also reflect structural changes upon ATP binding (Halawani et al. 2009). Moreover, mutations in the VCP gene may result in the disruption of normal VCP function leading to diffuse intracellular accumulation of ubiquitinated proteins that may contribute to the disease pathogenesis.

Our present studies and reports from other groups indicate that despite exhibiting reduced ATPase activity, the K524A mutant functions similarly to the clinical mutants by perturbing normal VCP functions and 

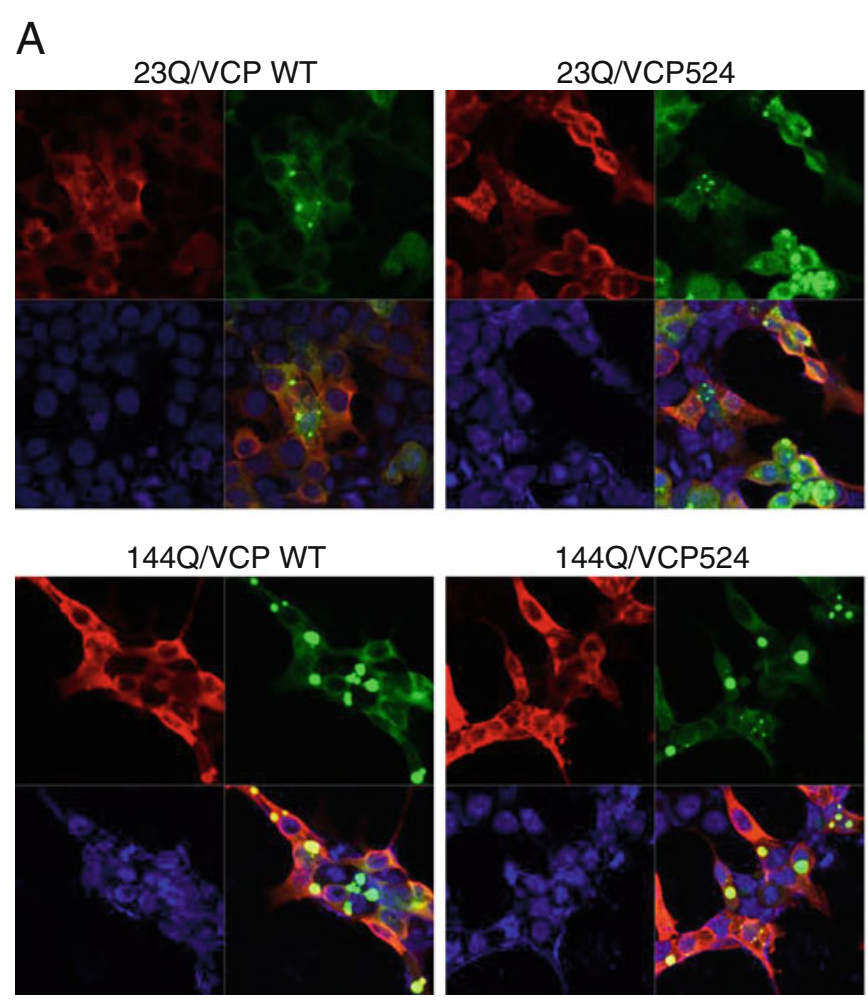

B

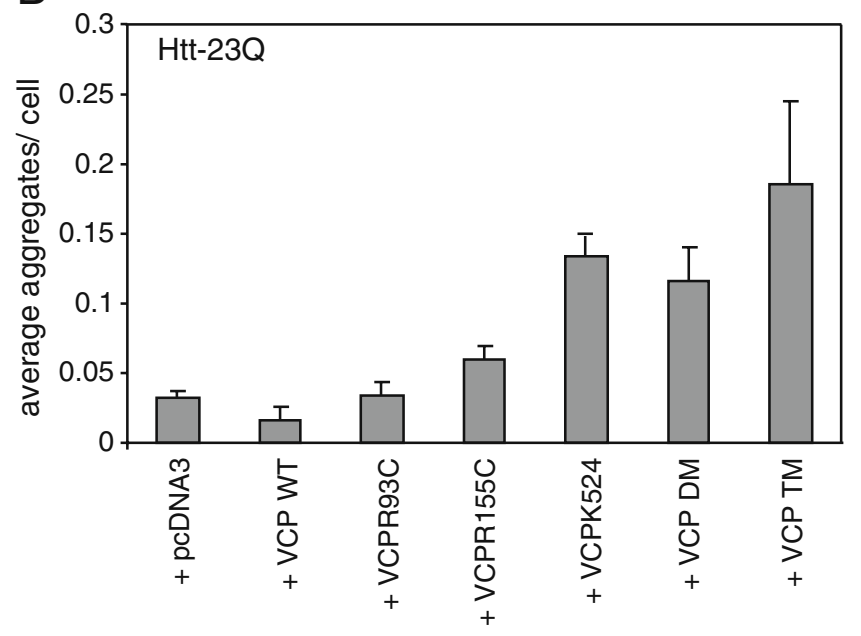

Fig. 4 Analysis by confocal microscopy of GFP-Htt aggregation after co-transfection with VCP constructs. a Following transfection of 293 T cells with VCPWT, K524A, DM (R93C, R155C) or TM (R93C, R155C, K524A) cDNA construct together with Htt-23Q or Htt-144Q, cells were permeabilized as mentioned in "Experimental Procedures" and serial stained first for Huntington protein and then for VCP as described in "Experimental Procedures". Confocal images were acquired using a Zeiss LSM510 NLO with Argon $488 \mathrm{~nm}, 543 \mathrm{~nm}$ $\mathrm{HeNe}$ and Coherent Chameleon XR 2p laser with appropriate

triggering abnormal protein aggregation and accumulation leading to increased cell death (Hirabayashi et al. 2001; Kobayashi et al. 2002; Manno et al. 2010). This suggests that the substrate binding to the $\mathrm{N}$-terminal
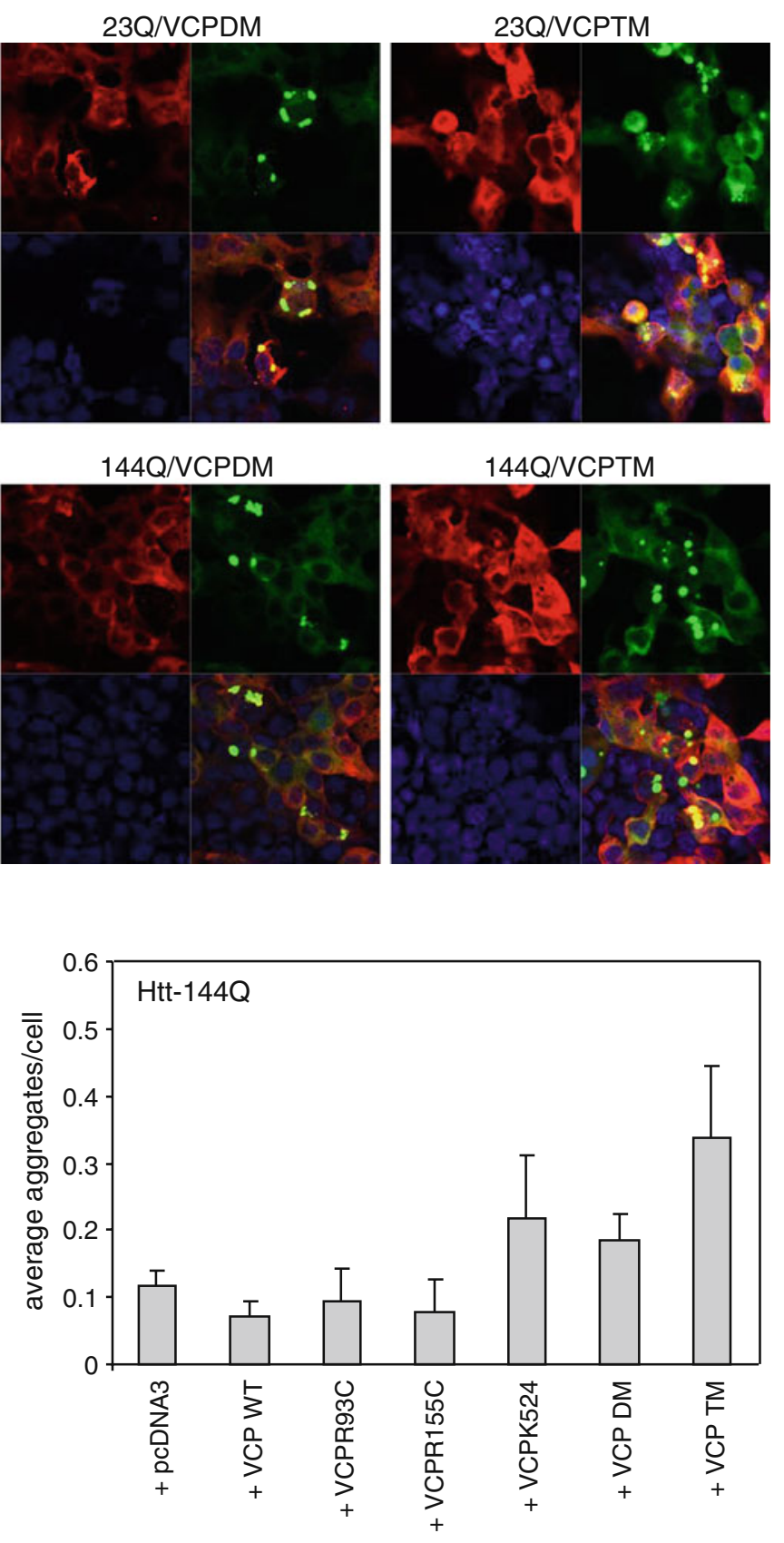

emission filters. b Quantification of Htt aggregates in VCPWT or VCP mutant-expressing cells. Htt aggregates were counted with Bitplane Imaris 6.4 using the Spots module as described in "Experimental Procedures". From the GFP-positive cells in a given field, total number of aggregates were counted and averaged among the three replicate fields containing at least 300 cells/sample. Units are expressed as the average number of aggregates/cell and are determined by quantifying the average number of GFP aggregates per GFP-positive cells

portion of VCP mutants may affect its D2-ATPase activity and function similarly to the VCP K524A or TM (R93C/ $\mathrm{R} 155 \mathrm{C} / \mathrm{K} 524 \mathrm{~A}$ ) which may then interfere with ubiquitindependent pathways thereby leading to abnormal protein 
aggregation in the nucleus and cytoplasm as well as enhanced cell death. Interestingly, NSF(K549A), an NSF mutant containing an equivalent mutation to $\mathrm{VCP}$ (K524A), has been reported to lose its ATPase activity and ATP binding and has been proposed to function in a dominant-negative manner within the NSF hexameric complex (Whiteheart et al. 1994).

Transgenic mice expressing the IBMPFD clinically relevant VCP mutant forms develop abnormal muscle pathology including coarse internal architecture, vacuolation, and disorganized membrane morphology with reduced caveolin-3 expression at the sarcolemma (Weihl et al. 2007; Custer et al. 2010). These animals also display an increase in ubiquitin-containing protein inclusions and high molecular weight ubiquitinated proteins suggesting that the early and persistent increase in ubiquitinated proteins induced by IBMPFD mutations in p97/VCP may ultimately lead to animal weakness and the observed muscle pathology (Weihl et al. 2007; Custer et al. 2010). The in vivo effects are thus hypothesized to be due to an accumulation of misfolded proteins such that, in neuronal tissues with a lower rate of turnover, the effect would be degeneration (such as FTD), whereas surviving cells would feature accumulated misfolded proteins and thus inclusion bodies (such as IBM). It would be interesting to understand if more noticeable phenotypes could be uncovered if mutant VCP were to replace endogenous VCP with the rationale being that there would be a greater likelihood of seeing more profound effects of the FTD mutants if endogenous VCP were knocked down. It is also possible that future studies examining parameters other than the ones we chose to study in this work or using other model systems could uncover effects of the clinically relevant VCP mutants.

Acknowledgments We thank Drs Juliette Gafni and Lisa M. Ellerby (Buck Institute for Research on Aging) for the Htt-23Q and Htt-144Q cDNA, Dr. A. Kakizuka (The Fourth Department, Osaka Bioscience Institute, Osaka 565-0874, Japan) for the VCPWT and VCPK524A cDNA, members of the Bredesen laboratory for helpful comments and discussions and Molly Susag for administrative assistance. This work was supported by grants from the National Institutes of Health NS33376 to D.E.B. \& R.V.R and AG034427-02 to DEB.

Open Access This article is distributed under the terms of the Creative Commons Attribution Noncommercial License which permits any noncommercial use, distribution, and reproduction in any medium, provided the original author(s) and source are credited.

\section{References}

Bence NF, Sampat RM, Kopito RR (2001) Impairment of the ubiquitin-proteasome system by protein aggregation. Science 292:1552-1555
Bence NF, Bennett EJ, Kopito RR (2005) Application and analysis of the GFPu family of ubiquitin-proteasome system reporters. Methods Enzymol 399:481-490

Boeddrich A, Gaumer S, Haacke A, Tzvetkov N, Albrecht M, Evert $\mathrm{BO}$ et al (2006) An arginine/lysine-rich motif is crucial for VCP/ p97-mediated modulation of ataxin-3 fibrillogenesis. Embo $\mathrm{J}$ 25:1547-1558

Bredesen DE, Rao RV, Mehlen P (2006) Cell death in the nervous system. Nature 443:796-802

Chen L, Gao X (2002) Neuronal apoptosis induced by endoplasmic reticulum stress. Neurochem Res 27:891-898

Custer SK, Neumann M, Lu H, Wright AC, Taylor JP (2010) Transgenic mice expressing mutant forms $\mathrm{VCP} / \mathrm{p} 97$ recapitulate the full spectrum of IBMPFD including degeneration in muscle, brain and bone. Hum Mol Genet 19:1741-1755

Dai RM, Li CC (2001) Valosin-containing protein is a multi-ubiquitin chain-targeting factor required in ubiquitin-proteasome degradation. Nat Cell Biol 3:740-744

Dai RM, Chen E, Longo DL, Gorbea CM, Li CC (1998) Involvement of valosin-containing protein, an ATPase co-purified with IkappaBalpha and $26 \mathrm{~S}$ proteasome, in ubiquitin-proteasomemediated degradation of IkappaBalpha. J Biol Chem 273:35623573

Doss-Pepe EW, Stenroos ES, Johnson WG, Madura K (2003) Ataxin3 interactions with $\operatorname{rad} 23$ and valosin-containing protein and its associations with ubiquitin chains and the proteasome are consistent with a role in ubiquitin-mediated proteolysis. Mol Cell Biol 23:6469-6483

Egerton M, Ashe OR, Chen D, Druker BJ, Burgess WH, Samelson LE (1992) VCP, the mammalian homolog of cdc48, is tyrosine phosphorylated in response to $\mathrm{T}$ cell antigen receptor activation. Embo J 11:3533-3540

Egger L, Madden DT, Rheme C, Rao RV, Bredesen DE (2007) Endoplasmic reticulum stress-induced cell death mediated by the proteasome. Cell Death Differ 14:1172-1180

Forman MS, Lee VM, Trojanowski JQ (2003) 'Unfolding' pathways in neurodegenerative disease. Trends Neurosci 26:407410

Forman MS, Mackenzie IR, Cairns NJ, Swanson E, Boyer PJ, Drachman DA et al (2006) Novel ubiquitin neuropathology in frontotemporal dementia with valosin-containing protein gene mutations. J Neuropathol Exp Neurol 65:571-581

Gitcho MA, Strider J, Carter D, Taylor-Reinwald L, Forman MS, Goate AM et al (2009) VCP mutations causing frontotemporal lobar degeneration disrupt localization of TDP-43 and induce cell death. J Biol Chem 284:12384-12398

Guyant-Marechal L, Laquerriere A, Duyckaerts C, Dumanchin C, Bou J, Dugny F et al (2006) Valosin-containing protein gene mutations: clinical and neuropathologic features. Neurology 67:644-651

Halawani D, LeBlanc AC, Rouiller I, Michnick SW, Servant MJ, Latterich M (2009) Hereditary inclusion body myopathy-linked p97/VCP mutations in the NH2 domain and the D1 ring modulate p97/VCP ATPase activity and D2 ring conformation. Mol Cell Biol 29:4484-4494

Hashimoto M, Rockenstein E, Crews L, Masliah E (2003) Role of protein aggregation in mitochondrial dysfunction and neurodegeneration in Alzheimer's and Parkinson's diseases. Neuromolecular Med 4:21-36

Higashiyama H, Hirose F, Yamaguchi M, Inoue YH, Fujikake N, Matsukage A et al (2002) Identification of ter94, Drosophila $\mathrm{VCP}$, as a modulator of polyglutamine-induced neurodegeneration. Cell Death Differ 9:264-273

Hirabayashi M, Inoue K, Tanaka K, Nakadate K, Ohsawa Y, Kamei Y et al (2001) VCP/p97 in abnormal protein aggregates, cytoplas- 
mic vacuoles, and cell death, phenotypes relevant to neurodegeneration. Cell Death Differ 8:977-984

Hubbers CU, Clemen CS, Kesper K, Boddrich A, Hofmann A, Kamarainen $\mathrm{O}$ et al (2007) Pathological consequences of VCP mutations on human striated muscle. Brain 130:381-393

Igarashi S, Morita H, Bennett KM, Tanaka Y, Engelender S, Peters MF et al (2003) Inducible PC12 cell model of Huntington's disease shows toxicity and decreased histone acetylation. Neuroreport 14:565-568

Ju JS, Miller SE, Hanson PI, Weihl CC (2008) Impaired protein aggregate handling and clearance underlie the pathogenesis of p97/VCP-associated disease. J Biol Chem 283:30289-30299

Ju JS, Fuentealba RA, Miller SE, Jackson E, Piwnica-Worms D, Baloh RH et al (2009) Valosin-containing protein (VCP) is required for autophagy and is disrupted in VCP disease. J Cell Biol 187:875-888

Katayama T, Imaizumi K, Manabe T, Hitomi J, Kudo T, Tohyama M (2004) Induction of neuronal death by ER stress in Alzheimer's disease. J Chem Neuroanat 28:67-78

Kimonis VE, Fulchiero E, Vesa J, Watts G (2008) VCP disease associated with myopathy, Paget disease of bone and frontotemporal dementia: review of a unique disorder. Biochim Biophys Acta $1782: 744-748$

Kobayashi T, Tanaka K, Inoue K, Kakizuka A (2002) Functional ATPase activity of p97/valosin-containing protein (VCP) is required for the quality control of endoplasmic reticulum in neuronally differentiated mammalian PC12 cells. J Biol Chem 277:47358-47365

Kopito RR (2000) Aggresomes, inclusion bodies and protein aggregation. Trends Cell Biol 10:524-530

Lindholm D, Wootz H, Korhonen L (2006) ER stress and neurodegenerative diseases. Cell Death Differ 13:385-392

Manno A, Noguchi M, Fukushi J, Motohashi Y, Kakizuka A (2010) Enhanced ATPase activities as a primary defect of mutant valosin-containing proteins that cause inclusion body myopathy associated with Paget disease of bone and frontotemporal dementia. Genes Cells 15:911-922

Mimnaugh EG, Xu W, Vos M, Yuan X, Neckers L (2006) Endoplasmic reticulum vacuolization and valosin-containing protein relocalization result from simultaneous hsp90 inhibition by geldanamycin and proteasome inhibition by velcade. Mol Cancer Res 4:667-681

Mishra M, Paunesku T, Woloschak GE, Siddique T, Zhu LJ, Lin S et al (2007) Gene expression analysis of frontotemporal lobar degeneration of the motor neuron disease type with ubiquitinated inclusions. Acta Neuropathol 114:81-94

Pleasure IT, Black MM, Keen JH (1993) Valosin-containing protein, VCP, is a ubiquitous clathrin-binding protein. Nature 365:459-462

Rao RV, Bredesen DE (2004) Misfolded proteins, endoplasmic reticulum stress and neurodegeneration. Curr Opin Cell Biol 16:653-662

Rao RV, Hermel E, Castro-Obregon S, del Rio G, Ellerby LM, Ellerby $\mathrm{HM}$ et al (2001) Coupling endoplasmic reticulum stress to the cell death program. Mechanism of caspase activation. J Biol Chem 276:33869-33874

Rao RV, Peel A, Logvinova A, del Rio G, Hermel E, Yokota T et al (2002) Coupling endoplasmic reticulum stress to the cell death program: role of the ER chaperone GRP78. FEBS Lett 514:122-128

Rao RV, Ellerby HM, Bredesen DE (2004a) Coupling endoplasmic reticulum stress to the cell death program. Cell Death Differ 11:372-380

Rao RV, Poksay KS, Castro-Obregon S, Schilling B, Row RH, Del Rio G et al (2004b) Molecular components of a cell death pathway activated by endoplasmic reticulum stress. J Biol Chem 279:177-187

Rao RV, Niazi K, Mollahan P, Mao X, Crippen D, Poksay KS et al (2006) Coupling endoplasmic reticulum stress to the cell-death program: a novel HSP90-independent role for the small chaperone protein p23. Cell Death Differ 13:415-425

Schilling B, Gafni J, Torcassi C, Cong X, Row RH, LaFevre-Bernt MA et al (2006) Huntingtin phosphorylation sites mapped by mass spectrometry. Modulation of cleavage and toxicity. J Biol Chem 281:23686-23697

Schroder R, Watts GD, Mehta SG, Evert BO, Broich P, Fliessbach K et al (2005) Mutant valosin-containing protein causes a novel type of frontotemporal dementia. Ann Neurol 57:457-461

Seki T, Takahashi H, Adachi N, Abe N, Shimahara T, Saito N et al (2007) Aggregate formation of mutant protein kinase $\mathrm{C}$ gamma found in spinocerebellar ataxia type 14 impairs ubiquitinproteasome system and induces endoplasmic reticulum stress. Eur J Neurosci 26:3126-3140

Song C, Wang Q, Li CC (2003) ATPase activity of p97-valosincontaining protein (VCP). D2 mediates the major enzyme activity, and D1 contributes to the heat-induced activity. J Biol Chem 278:3648-3655

Tresse E, Salomons FA, Vesa J, Bott LC, Kimonis V, Yao TP et al (2010) $\mathrm{VCP} / \mathrm{p} 97$ is essential for maturation of ubiquitin-containing autophagosomes and this function is impaired by mutations that cause IBMPFD. Autophagy 6

Vale RD (2000) AAA proteins. Lords of the ring J Cell Biol 150:F13F19

Vandermoere F, El Yazidi-Belkoura I, Slomianny C, Demont Y, Bidaux G, Adriaenssens E et al (2006) The valosin-containing protein (VCP) is a target of Akt signaling required for cell survival. J Biol Chem 281:14307-14313

Vesa J, Su H, Watts GD, Krause S, Walter MC, Martin B et al (2009) Valosin containing protein associated inclusion body myopathy: abnormal vacuolization, autophagy and cell fusion in myoblasts. Neuromuscul Disord 19:766-772

Vij N (2008) AAA ATPase p97/VCP: cellular functions, disease and therapeutic potential. J Cell Mol Med 12:2511-2518

Wang Q, Song C, Yang X, Li CC (2003) D1 ring is stable and nucleotide-independent, whereas D2 ring undergoes major conformational changes during the ATPase cycle of p97-VCP. J Biol Chem 278:32784-32793

Watts GD, Wymer J, Kovach MJ, Mehta SG, Mumm S, Darvish D et al (2004) Inclusion body myopathy associated with Paget disease of bone and frontotemporal dementia is caused by mutant valosin-containing protein. Nat Genet 36:377-381

Weihl CC, Dalal S, Pestronk A, Hanson PI (2006) Inclusion body myopathy-associated mutations in p97/VCP impair endoplasmic reticulum-associated degradation. Hum Mol Genet 15:189199

Weihl CC, Miller SE, Hanson PI, Pestronk A (2007) Transgenic expression of inclusion body myopathy associated mutant $\mathrm{p} 97 /$ VCP causes weakness and ubiquitinated protein inclusions in mice. Hum Mol Genet 16:919-928

Weihl CC, Pestronk A, Kimonis VE (2009) Valosin-containing protein disease: inclusion body myopathy with Paget's disease of the bone and fronto-temporal dementia. Neuromuscul Disord 19:308-315

Whiteheart SW, Rossnagel K, Buhrow SA, Brunner M, Jaenicke R, Rothman JE (1994) N-ethylmaleimide-sensitive fusion protein: a trimeric ATPase whose hydrolysis of ATP is required for membrane fusion. J Cell Biol 126:945-954

Wojcik C (2002) VCP - the missing link in protein degradation? Trends Cell Biol 12:212

Xu C, Bailly-Maitre B, Reed JC (2005) Endoplasmic reticulum stress: cell life and death decisions. J Clin Invest 115:2656-2664

Yang H, Liu C, Zhong Y, Luo S, Monteiro MJ, Fang S (2010) Huntingtin interacts with the cue domain of gp78 and inhibits gp78 binding to ubiquitin and p97/VCP. PLoS One 5:e8905 
Yoshida H (2007) ER stress and diseases. FEBS J 274:630-658

Zhang K, Kaufman RJ (2006) The unfolded protein response: a stress signaling pathway critical for health and disease. Neurology 66: S102-S109

Zhong X, Pittman RN (2006) Ataxin-3 binds VCP/p97 and regulates retrotranslocation of ERAD substrates. Hum Mol Genet $15: 2409-2420$
After acceptance of this manuscript, Johnson et al (Neuron; 68, $857-$ 864 , December 9,2010 ) reported that VCP mutations may account for $1 \%-2 \%$ of familial ALS. Their findings further suggest that VCP mutations may lead to the accumulation of degraded proteins observed as ubiquitinated inclusions thus implicating the role of VCP in the ubiquitination/protein degradation pathway in diseases that feature misfolded proteins including (but not limited to) FTD, ALS and HD. 